\title{
Switch-Associated Protein 70 Antibodies in Multiple Sclerosis: Possible Association with Disease Progression
}

\author{
Recai Türkoğlu ${ }^{a}$ Mehmet Gencer ${ }^{a}$ Duygu Ekmekçia Canan Ulusoy ${ }^{b}$

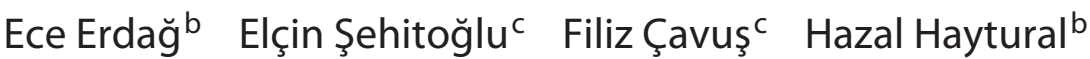 \\ Melike Küçükerden ${ }^{b}$ Nazlı Yalçınkaya ${ }^{b}$ Selin Turan ${ }^{b}$ Deniz Akbaş-Demir ${ }^{a}$ \\ Arzu Çoban ${ }^{b}$ Burçak Vural ${ }^{c}$ Erdem Tüzün ${ }^{b}$ \\ ${ }^{a}$ Department of Neurology, Haydarpasa Numune Education and Research Hospital, and Departments of \\ ${ }^{b}$ Neuroscience and ${ }^{\mathrm{C}}$ Genetics, Institute for Experimental Medicine (DETAE), Istanbul University, Istanbul, Turkey
}

\section{Key Words}

Antibody · Chemokine - Multiple sclerosis · Relapse ·

Switch-associated protein 70

\section{Abstract}

Objective: This study was conducted to identify a biomarker for multiple sclerosis (MS) that can be used as a predictor of relapse and disability. Materials and Methods: Sera of 26 consecutive relapsing-remitting MS (RRMS) patients were screened for switch-associated protein 70 (SWAP-70) antibody, which was previously identified by protein macroarray. The serum levels of several cytokines, chemokines and soluble adhesion molecules related to MS attacks were measured by enzyme-linked immunosorbent assay (ELISA). A possible correlation was sought among levels of SWAP-70 antibody, measured humoral factors and disability scores. Results: ELISA studies showed high-titre SWAP-70 antibodies in $16(61.5 \%)$ RRMS sera obtained during the attack period and 9 (34.6\%) sera obtained during remission. There was a significant inverse correlation between SWAP-70 antibody levels and expanded disability status scale scores, CXCL10, soluble VCAM-1, CXCL13 and soluble VLA-4 levels. Conclu- sion: Our results showed that SWAP-70 antibodies could potentially be utilized as relapse and prognostic biomarkers in MS. Whether or not SWAP-70 antibodies have any effect on disease mechanisms requires further investigation.

(c) 2014 S. Karger AG, Basel

\section{Introduction}

Multiple sclerosis (MS) is a chronic demyelinating and neurodegenerative disease of the central nervous system. While myelin-reactive $\mathrm{T}$ cells are primary mediators in MS pathogenesis, $B$ cells and humoral factors are also crucially involved $[1,2]$. The presence of intrathecal IgG production and IgG deposits in MS lesions and the response to antibody-depleting treatment methods suggest that antibodies might modulate the clinical progression of MS [1].

Recently, we identified the switch-associated protein 70 (SWAP-70) antibody in the sera of relapsing-remitting MS (RRMS) patients [3]. This antibody was mostly detected during or shortly after relapse, and its serum levels were inversely correlated with the expanded disability 
status scale (EDSS) scores of the patients [3]. SWAP-70 is highly expressed in lymphoid organs and participates in lymphocyte activation and migration as well as vascular cell adhesion [4-6]. Humoral factors related to MS pathogenesis and SWAP-70 functions have been previously characterized [7-13]. Hence, the objective of this study was to investigate whether the association between SWAP-70 antibody levels and disability scores could be due to an altered production of these humoral factors. The levels of several cytokines, chemokines and soluble adhesion molecules were measured in MS patients' sera during and after an attack, and a possible correlation between the levels of these factors, antibody levels and clinical features was investigated.

\section{Materials and Methods}

\section{Patients and Samples}

Twenty-six consecutive RRMS patients (19 women and 7 men; age range $24-60$ years) who were admitted to our outpatient clinic with an MS attack and 50 age- and gender-matched healthy controls (36 women and 14 men; age range $27-58$ years) were included in this study. All patients had definite MS according to McDonald criteria [14], had no other autoimmune or infectious diseases and were negative for anti-nuclear antibody (ANA). MS durations ranged from 1 to 36 years, and EDSS scores ranged between 1.0 and 5.0. All patients were under treatment with a disease-modifying drug (interferon- $\beta, n=14$; glatiramer acetate, $n=5$; fingolimod, $\mathrm{n}=4$, and natalizumab, $\mathrm{n}=3$ ). All patients received pulse methylprednisolone treatment during the attacks. The attack sera were obtained prior to steroid treatment. Serum samples were collected from all RRMS patients during relapse and remission periods ( 3 months after relapse in all patients). None of the patients had another MS attack within 4 months prior to the first blood sampling or during the study period. All sera were kept frozen at $-80^{\circ} \mathrm{C}$ until assayed. This study was approved by the Ethics Committee of the Istanbul Faculty of Medicine.

Protein Expression

High-titre antibodies to SWAP-70 [accession No. NM_015055; National Center for Biotechnology Information (NCBI), Bethesda, Md., USA], heat shock protein 70 (HSP-70) (NCBI accession No. NM_002154) and inhibitor of growth family, member 4 (ING4) (NCBI accession No. NM_016162) were previously identified in MS patients' sera using a protein macroarray derived from the human fetal brain cDNA expression library (hEX1) (ImaGenes, Berlin, Germany) $[3,15,16]$. The plasmid DNA of these autoantigens was isolated and sequenced. Nucleotide and translated amino acid sequences were compared with known sequences using BLAST algorithms (NCBI). His-tagged proteins were then recombinantly expressed in Escherichia coli and purified by affinity chromatography, and the purity of the protein was documented by SDS-PAGE analysis and immunoblot experiments using commercial antibodies against SWAP-70, HSP-70 and ING4 (Abcam, San Francisco, Calif., USA) and the sera of patients and controls, as previously described $[3,16]$.
Enzyme-Linked Immunosorbent Assay for Antibodies

Detection of serum antibodies to the purified recombinant human proteins extracted from E. coli as well as the lysate of the same E. coli strain with no human protein expression was performed with an enzyme-linked immunosorbent assay (ELISA) using the sera of all patients and healthy controls, as reported previously [3, $15,16]$.

\section{ELISA for Cytokines, Chemokines and Soluble Adhesion}

Molecules

Serum human soluble very late antigen-4 (sVLA-4), CCL5, CXCL13 (Cusabio, Wuhan, China), IL-6, IL-8, IL-13, IL-17A, monocyte chemotactic protein-1 (MCP1), CXCL10, soluble vascular cell adhesion molecule 1 (sVCAM-1) and soluble intercellular adhesion molecule 1 (sICAM-1) levels were measured using ELISA kits (Diaclone, Canton, Mass., USA) according to the manufacturer's instructions. Optical density was measured at $450 \mathrm{~nm}$, and concentrations were calculated by referring to a standard curve.

\section{Statistics}

Due to an abnormal distribution of the data (assessed by the Kolmogorov-Smirnov test) in most groups, statistical comparisons between subgroups were performed using the Mann-Whitney U test or Fisher's exact test, as required. Comparisons between serum antibody levels were done with the Kruskal-Wallis test and Dunn's post hoc test. Correlation statistics were performed with Pearson's or Spearman's correlation test, as appropriate. $\mathrm{p}<0.05$ was considered statistically significant.

\section{Results}

\section{Association between SWAP-70 Antibody Levels and Clinical Features}

Among the 26 RRMS patients, SWAP-70, HSP-70 and ING4 antibodies were detected in the attack and/or remission sera of $16(61.5 \%), 23(88.5 \%)$ and $19(73.1 \%)$ patients, respectively. None of the healthy controls had antibodies to these proteins and none of the MS patients had high-titre antibodies (above 2 SD from the healthy control average) to the non-human protein expressing $E$. coli lysate (fig. 1a). In immunoblotting experiments, both the commercial SWAP-70 antibody and SWAP-70 antibody-positive sera but not negative samples bound a single band at $70 \mathrm{kDa}$, indicating the specificity of these antibodies (data not shown). Compared to healthy controls, the levels of SWAP-70, HSP-70 and ING4 but not E. coli lysate antibodies were significantly higher in MS patients during both attack and remission periods $(\mathrm{p}<0.05$ by the Kruskal-Wallis test and Dunn's post hoc test; fig. 1a). In contrast, the prevalence of SWAP-70 antibody seropositivity was found to be significantly higher during attacks (16 patients) than during remission periods (9 patients) ( $p=0.047$ by Fisher's exact test). Seropositivity rates were 

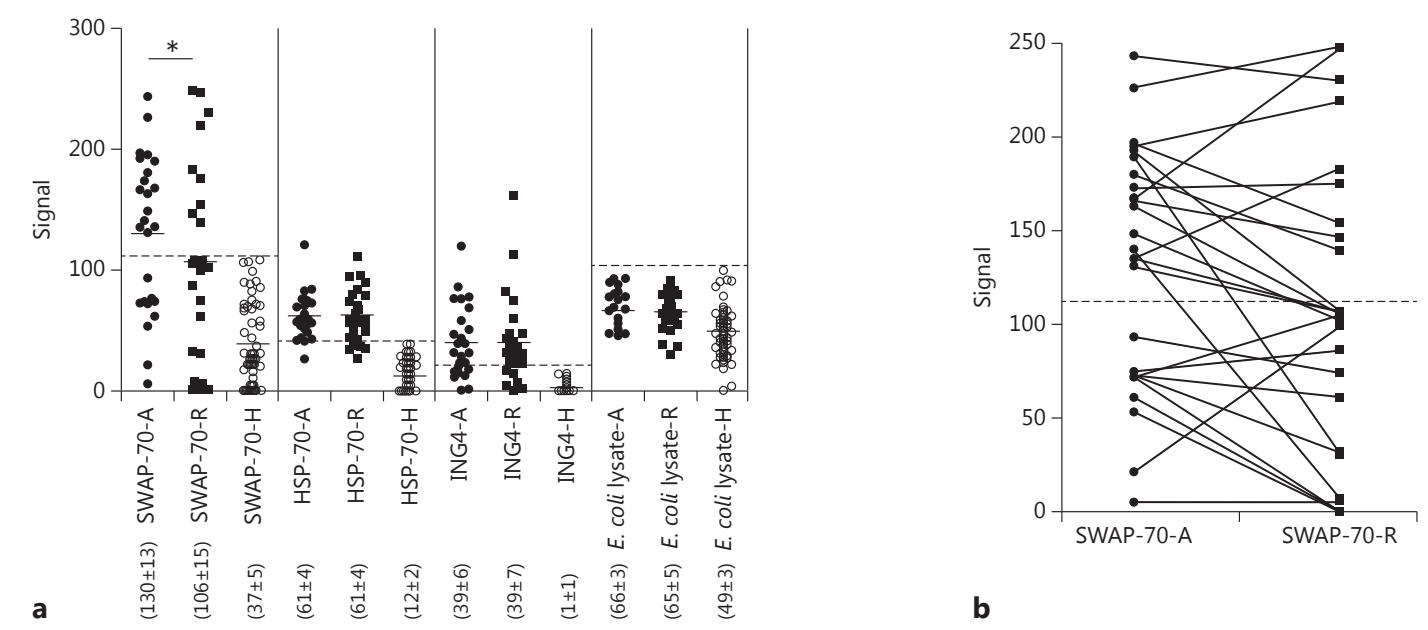

Fig. 1. a ELISA detection of IgG antibodies (fluorescence signal values) to SWAP-70, HSP-70, ING4 and the E. coli lysate in the sera of healthy controls $(\mathrm{H})$ and relapsing RRMS patients during attack (A) and remission (R) periods. Horizontal lines indicate the mean values of each group, and the numbers in parentheses indi-

cate the average \pm SE for each group. ${ }^{*} \mathrm{p}<0.05$. $\mathbf{b}$ Comparison of serum SWAP-70 antibody levels of RRMS patients during attack (A) and remission (R) periods. The dashed lines represent the cutoff value for SWAP-70 antibody seropositivity (2 SD above the mean of healthy controls).

Table 1. Demographic and clinical features of SWAP-70 antibody-positive and SWAP-70 antibody-negative MS patients during attack and remission periods

\begin{tabular}{|c|c|c|c|c|c|c|}
\hline & \multicolumn{3}{|l|}{ Attack } & \multicolumn{3}{|l|}{ Remission } \\
\hline & $\begin{array}{l}\text { SWAP-70 Ab+ } \\
(\mathrm{n}=16)\end{array}$ & $\begin{array}{l}\text { SWAP-70 Ab- } \\
(\mathrm{n}=10)\end{array}$ & $\begin{array}{l}\mathrm{p} \\
\text { value }\end{array}$ & $\begin{array}{l}\text { SWAP-70 Ab+ } \\
(\mathrm{n}=9)\end{array}$ & $\begin{array}{l}\text { SWAP-70 Ab- } \\
(\mathrm{n}=17)\end{array}$ & $\begin{array}{l}\mathrm{p} \\
\text { value }\end{array}$ \\
\hline Female/male ratio & $9 / 7$ & $10 / 0$ & $0.023^{1}$ & $5 / 4$ & $14 / 3$ & $0.188^{1}$ \\
\hline Age, years & $39.4 \pm 2.6$ & $40.0 \pm 4.4$ & $0.336^{2}$ & $38.0 \pm 2.2$ & $40.5 \pm 4.1$ & $1.000^{2}$ \\
\hline Duration of MS, years & $9.2 \pm 2.2$ & $6.8 \pm 0.9$ & $0.421^{2}$ & $9.5 \pm 2.8$ & $7.7 \pm 1.5$ & $1.000^{2}$ \\
\hline \multicolumn{7}{|l|}{$\mathrm{DMD}, \mathrm{n}$} \\
\hline Interferon- $\beta$ & 7 & 7 & $0.247^{1}$ & 4 & 10 & $0.683^{1}$ \\
\hline Glatiramer acetate & 3 & 2 & $1.000^{1}$ & 1 & 4 & $0.628^{1}$ \\
\hline Others & 6 & 1 & $0.191^{1}$ & 4 & 3 & $0.188^{1}$ \\
\hline
\end{tabular}

Values are presented as means \pm SE unless otherwise specified. $\mathrm{Ab}=$ Antibody; $\mathrm{DMD}=$ disease-modifying drug. ${ }^{1}$ Fisher's exact test. ${ }^{2}$ Mann-Whitney U test.

identical for other antibodies during attack and remission (HSP-70 antibody: 23 vs. 21 patients, $\mathrm{p}=0.351$, and ING4 antibody: 19 vs. 18 patients, $\mathrm{p}=0.500$ ). Also, the serum levels of SWAP-70 antibodies were significantly higher in attack sera (average 130; range 5-243) than in remission sera (average 106; range $0-248$ ) ( $\mathrm{p}=0.046$ by the MannWhitney U test) (fig. 1a). While 7 of the 16 SWAP-70 antibody-positive MS patients became seronegative during the remission period, none of the SWAP-70 antibodynegative patients seroconverted (fig. 1b). 


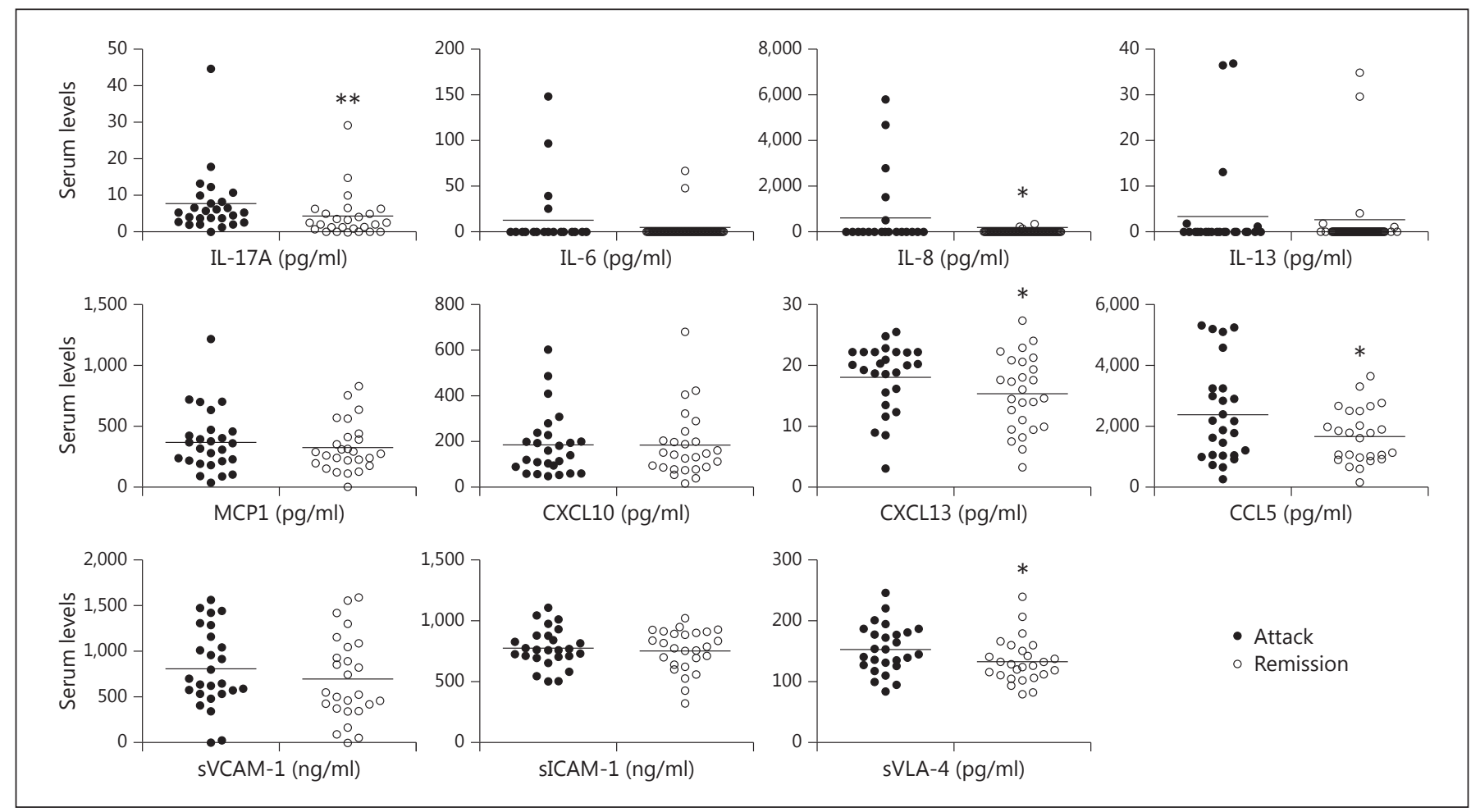

Fig. 2. Serum cytokine, chemokine and soluble adhesion molecule levels of RRMS patients during attack and remission periods. Horizontal lines indicate mean values. ${ }^{*} \mathrm{p}<0.05,{ }^{* *} \mathrm{p}<0.01$.

There were no significant differences between the clinical and demographic features of SWAP-70 antibodypositive and SWAP-70 antibody-negative patients, with the exception that the female-to-male ratio was significantly higher in the seronegative group during the attack period ( $p=0.023$ by Fisher's exact test). Also, seropositive patients showed trends towards exhibiting lower annual attack numbers and EDSS scores (table 1). Moreover, there was a significant inverse correlation between EDSS scores and levels of SWAP-70 antibodies $(\mathrm{R}=-0.471, \mathrm{p}=$ 0.038 ) but not those of HSP-70, ING4 and E. coli lysate antibodies. There were no significant correlations between antibody levels and age, MS duration and annual attack number parameters.

Levels of Humoral Factors in SWAP-70 Antibody-

Positive and SWAP-70 Antibody-Negative MS Patients

In attack sera, statistically significant increases were observed in IL-17A ( $p=0.004)$, IL-8 ( $p=0.013)$, CXCL13 $(\mathrm{p}=0.042), \operatorname{CCL} 5(\mathrm{p}=0.045)$ and $\mathrm{sVLA}-4(\mathrm{p}=0.021)$ levels (fig. 2). SWAP-70 antibody-positive patients showed trends towards exhibiting lower serum levels of humoral factors during attack and remission periods. The only exception was IL-13, the levels of which were slightly but insignificantly $(\mathrm{p}=0.151)$ higher in the attack sera of seropositive patients (fig. 3). Likewise, IL-13 levels were significantly $(p=0.002)$ higher in the remission sera of seropositive patients (fig. 4). Additionally, serum CXCL13 levels were significantly $(p=0.033)$ reduced in seropositive patients during the remission period (fig. 4).

\section{Correlation between SWAP-70 Antibody and \\ Circulating Humoral Factor Levels}

During the attack period, SWAP-70 antibody levels showed a significant inverse correlation with only sVCAM-1 levels $(\mathrm{R}=-0.423, \mathrm{p}=0.018)$, whereas during the remission period, sVCAM-1 (R-0,344, $\mathrm{p}=0.043)$, CXCL10 ( $\mathrm{R}=-0.345, \mathrm{p}=0.042)$, CXCL13 $(\mathrm{R}=-0.493$, $\mathrm{p}=0.005)$ and sVLA $-4(\mathrm{R}=-0.367, \mathrm{p}=0.033)$ levels were inversely correlated and serum IL-13 levels $(\mathrm{R}=0.342$, $\mathrm{p}=0.044)$ were directly correlated with SWAP-70 antibody levels. The most significant correlations were obtained with CXCL13 levels in remission sera and sVCAM-1 levels in attack sera (fig. 5). No significant correlation could be established between the serum levels of other antibodies and circulating humoral factors. 


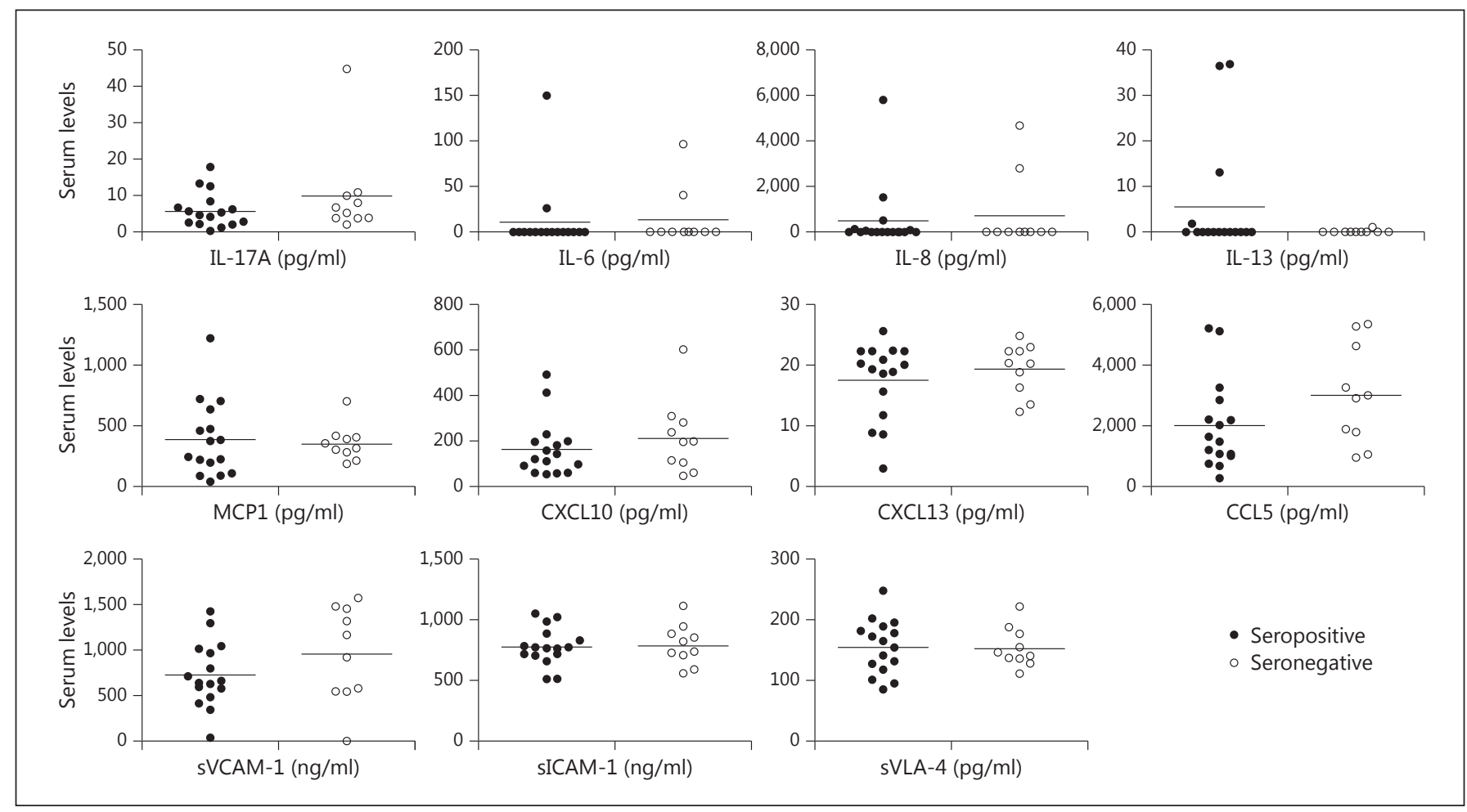

Fig. 3. Serum cytokine, chemokine and soluble adhesion molecule levels of SWAP-70 antibody-positive and SWAP-70 antibody-negative RRMS patients during the attack period. Horizontal lines indicate mean values.

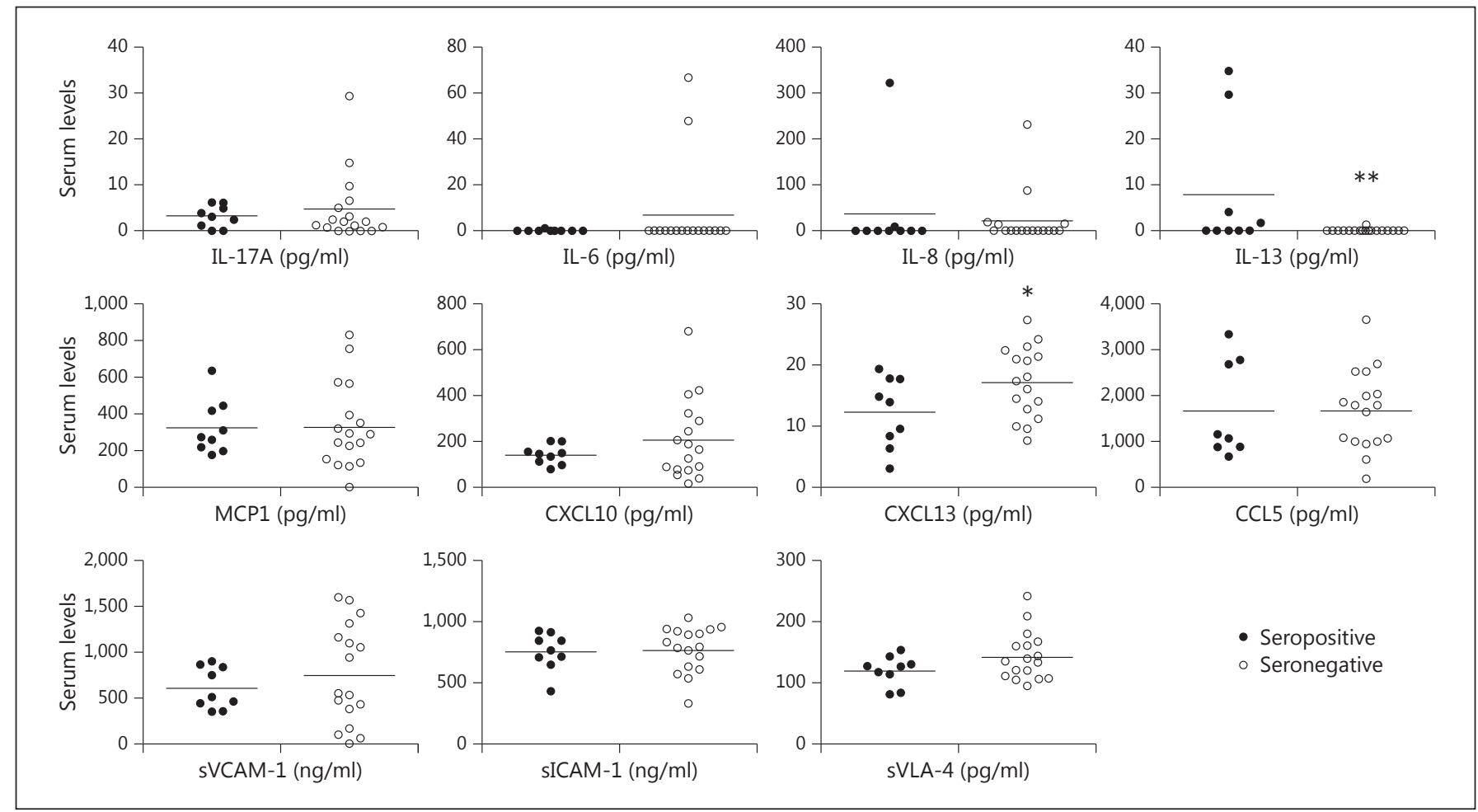

Fig. 4. Serum cytokine, chemokine and soluble adhesion molecule levels of SWAP-70 antibody-positive and SWAP-70 antibody-negative RRMS patients during the remission period. Horizontal lines indicate mean values. ${ }^{*} \mathrm{p}<0.05,{ }^{* *} \mathrm{p}<0.01$. 


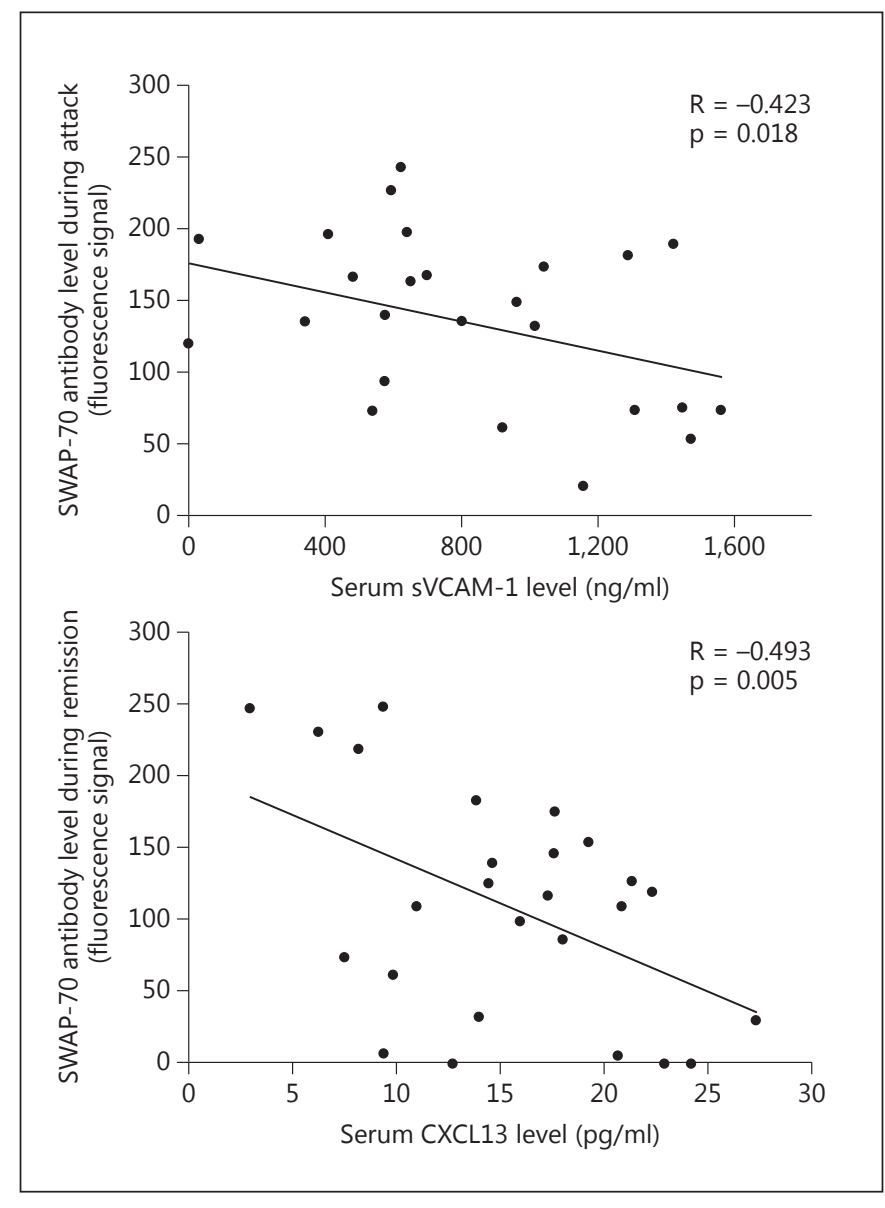

Fig. 5. Most significant correlations between serum levels of SWAP-70 antibody and humoral factors. p values by Pearson's correlation test.

\section{Discussion}

The present study confirms the results of our previous study [3], i.e. that SWAP-70 antibodies are found in MS patients with a relatively benign disease course and that their serum levels increase during MS attacks. These features indicate that the SWAP-70 antibody could have a potential role as an attack and disability biomarker in MS patients. Moreover, SWAP-70 antibody-positive patients showed relatively reduced levels of proinflammatory cytokine, chemokine and soluble adhesion molecules. CXCL13, CXCL10, sVCAM-1 and sVLA-4 levels were particularly lower in MS patients with high SWAP-70 antibody levels and vice versa. Importantly, levels of IL-13, which had previously been associated with a benign MS course and neuroprotective action in MS [12], were found to be significantly higher in SWAP-70 antibody-positive patients. No significant correlation was established between the levels of control antibodies and humoral factors, thereby giving further evidence that the presence of SWAP-70 antibodies is, albeit modestly, related to reduced levels of MS inducing proinflammatory humoral factors.

An intriguing question is whether SWAP-70 antibodies are simply passive byproducts of a common immunological mechanism that reduces MS severity or whether there is a causal relationship between SWAP-70 antibodies and reduced levels of proinflammatory serum factors. During MS attacks, SWAP-70 expression might plausibly increase in the lymphoid organs to compensate for the increased demand for lymphocyte maturation and migration. This might prompt the formation of SWAP-70 antibodies, which in turn would counteract the inflammatory mechanisms by suppressing SWAP-70 functions as a negative feedback mechanism. Somewhat identical antibody formation mechanisms have been described in systemic lupus erythematosus and myasthenia gravis patients, who develop C1q antibodies possibly in order to defuse complement deposition at the target tissue [17, 18]. Fingolimod is a member of the sphingosine-1-phosphate $(\mathrm{S} 1 \mathrm{P})$ receptor modulators, which appear to exert their effects at least partially via SWAP-70 inhibition [19, 20]. Notably, serum CCL5 and IL-17a levels were relatively reduced in both SWAP-70 antibody-positive and fingolimod-treated MS patients [21,22], further indicating a potential cytokine and chemokine modulating action for SWAP-70 antibodies.

\section{Conclusion}

Our results confirm that SWAP-70 antibody levels are elevated during MS attacks. Moreover, the inverse correlations between SWAP-70 antibody levels and EDSS scores and cytokine/chemokine levels suggest that SWAP-70 is involved in the MS attack pathogenesis. Overall, our findings suggest that SWAP-70 antibodies can potentially be utilized as relapse and prognosis biomarkers in MS.

\footnotetext{
References
1 Meinl E, Krumbholz M, Hohlfeld R: B lineage cells in the inflammatory central nervous sys- tem environment: migration, maintenance, local antibody production, and therapeutic modulation. Ann Neurol 2006;59:880-892. toimmune neuro-inflammation. Curr Opin Immunol 2011;23:707-712.

$\checkmark 2$ Becher B, Segal BM: T(H)17 cytokines in au-
} 
- Erdağ E, Tüzün E, Uğurel E, et al: Switch-associated protein 70 antibodies in multiple sclerosis: relationship between increased serum levels and clinical relapse. Inflamm Res 2012;61:927-930.

-4 Bahaie NS, Hosseinkhani MR, Ge XN, et al: Regulation of eosinophil trafficking by SWAP-70 and its role in allergic airway inflammation. J Immunol 2012;188:1479-1490.

5 Pearce G, Angeli V, Randolph GJ, et al: Signaling protein SWAP-70 is required for efficient B cell homing to lymphoid organs. Nat Immunol 2006;7:827-834.

-6 Sivalenka RR, Jessberger R: SWAP-70 regulates c-kit-induced mast cell activation, cellcell adhesion, and migration. Mol Cell Biol 2004;24:10277-10288.

7 Pigard N, Elovaara I, Kuusisto H, et al: Therapeutic activities of intravenous immunoglobulins in multiple sclerosis involve modulation of chemokine expression. J Neuroimmunol 2009;209:114-120.

8 Szczuciński A, Losy J: CCL5, CXCL10 and CXCL11 chemokines in patients with active and stable relapsing-remitting multiple sclerosis. Neuroimmunomodulation 2011; 18: 67-72.

-9 Baraczka K, Nékám K, Pozsonyi T, et al: Concentration of soluble adhesion molecules (sVCAM-1, sICAM-1 and sL-selectin) in the cerebrospinal fluid and serum of patients with multiple sclerosis and systemic lupus erythematosus with central nervous involvement. Neuroimmunomodulation 2001;9:49-54.
10 Alter A, Duddy M, Hebert S, et al: Determinants of human $B$ cell migration across brain endothelial cells. J Immunol 2003;170:44974505.

-11 Tachimoto H, Ebisawa M: Effect of interleukin-13 or tumor necrosis factor-alpha on eosinophil adhesion to endothelial cells under physiological flow conditions. Int Arch Allergy Immunol 2007;143:33-37.

12 Rossi S, Mancino R, Bergami A, et al: Potential role of IL-13 in neuroprotection and cortical excitability regulation in multiple sclerosis. Mult Scler 2011;17:1301-1312.

13 Kalinowska-Łyszczarz A, Szczuciński A, Pawlak MA, et al: Clinical study on CXCL13, CCL17, CCL20 and IL-17 as immune cell migration navigators in relapsing-remitting multiple sclerosis patients. J Neurol Sci 2011; 300:81-85.

14 Polman CH, Reingold SC, Edan G, et al: Diagnostic criteria for multiple sclerosis: 2005 revisions to the 'McDonald Criteria'. Ann Neurol 2005;58:840-846.

15 Vural B, Demirkan A, Ugurel E, et al: Seroreactivity against PTEN-induced putative kinase 1 (PINK1) in Turkish patients with Behçet's disease. Clin Exp Rheumatol 2009; 27:S67-S72.
16 Vural B, Uğurel E, Tüzün E, et al: Anti-neuronal and stress-induced-phosphoprotein 1 antibodies in neuro-Behçet's disease. J Neuroimmunol 2011;239:91-97.

17 Akhter E, Burlingame RW, Seaman AL, et al: Anti-C1q antibodies have higher correlation with flares of lupus nephritis than other serum markers. Lupus 2011;20:1267-1274.

18 Tüzün E, Saini SS, Ghosh S, et al: Predictive value of serum anti-C1q antibody levels in experimental autoimmune myasthenia gravis. Neuromuscul Disord 2006;16:137-143.

19 Ocaña-Morgner C, Reichardt P, Chopin M, et al: Sphingosine 1-phosphate-induced motility and endocytosis of dendritic cells is regulated by SWAP-70 through RhoA. J Immunol 2011;186:5345-5355.

20 Groves A, Kihara Y, Chun J: Fingolimod: direct CNS effects of sphingosine 1-phosphate (S1P) receptor modulation and implications in multiple sclerosis therapy. J Neurol Sci 2013;328:9-18.

21 Huu DL, Matsushita T, Jin G, et al: FTY720 ameliorates murine sclerodermatous chronic graft-versus-host disease by promoting expansion of splenic regulatory cells and inhibiting immune cell infiltration into skin. Arthritis Rheum 2013;65:1624-1635.

22 Nofer JR, Bot M, Brodde M, et al: FTY720, a synthetic sphingosine 1 phosphate analogue, inhibits development of atherosclerosis in low-density lipoprotein receptor-deficient mice. Circulation 2007;115:501-508. 